\title{
Creating methane from plastics: recycling at a lunar outpost
}

Janine Captain (KT-D1), Eddie Santiago (KT-D2), Ray Wheeler (KT-B) NASA KSC

Richard Strayer, Jay Garland, Dynamac, KSC

Clyde Parrish, C Parrish Consulting, New Port Richey, FL

The high cost of re-supply from Earth demands resources to be utilized to the fullest extent for exploration missions. Recycling is a key technology that maximizes the available resources by converting waste products into useful commodities. One example of this is to convert crew member waste such as plastic packaging, food scraps, and human waste, into fuel. The ability to refuel on the lunar surface would reduce the vehicle mass during launch and provide excess payload capability. The goal of this project is to determine the feasibility of recycling waste into methane on the lunar outpost by performing engineering assessments and lab demonstrations of the technology.

The first goal of the project was to determine how recycling could influence lunar exploration. Table I shows an estimation of the typical dried waste stream generated each day for a crew of four. Packaging waste accounts for nearly $86 \%$ of the dry waste stream and is a significant source of carbon on the lunar surface. This is important because methane $\left(\mathrm{CH}_{4}\right)$ can be used as fuel and no other source of carbon is available on the lunar surface. With the initial assessment indicating there is sufficient resources in the waste stream to provide refueling capabilities, the project was designed to examine the conversion of plastics into methane. Additional data on the analysis of the polymer resources can be found in Final Report Production of Methane and Water From Plastics and Crew Waste ${ }^{I}$.

Table I Elemental weights in grams for the daily packaging plastic waste, human dry solid waste, and dry food waste attached to plastic waste for a crew of four. ${ }^{1}$

\begin{tabular}{|l|c|c|c|c|c|c|c|}
\hline Waste Type & Total Wt. & C & H & O & N & S & Al \\
\hline Packaging waste & 1049 & 650 & 93 & 64 & 16 & 0 & 225 \\
\hline Human dry solid waste & 112 & 63 & 9 & 35 & 3 & 1 & 0 \\
\hline Dry food attached to plastic & 60 & 29 & 4 & 26 & 0 & 2 & 0 \\
\hline Total waste for crew of 4 & 1221 & 741 & 106 & 125 & 20 & 3 & 225 \\
\hline
\end{tabular}

The number listed in Table one come from the final report referenced above. Additional reports on crew requirements have larger values for these wastes ${ }^{2-3}$. These values make recycling the plastic packaging an even more compelling option for the production of methane.

The three main approaches examined for the degradation of the plastics were pyrolysis, gasification, and biological methods. Pyrolysis and gasification processes use high temperatures to degrade the plastics. While pyrolysis is performed under an inert atmosphere, gasification involves adding a controlled amount of oxygen during degradation. The products of both processes vary with temperature and the amount of oxygen added during gasification can also affect the products. The $\mathrm{CO}$ and $\mathrm{CO}_{2}$ produced during the degradation can be converted to methane using the Sabatier process.

The biological process refers to anaerobic digestion; hydrolysis, acetogenesis, and methanogenesis. Given the current architecture, the biological processing has a limited portion of the waste stream it can break down since the plastics are not biodegradable. However, if the plastics used during the missions were changed to biodegradable plastics, the entire waste stream could be utilized by the bacterial colonies. An investigational study into the availability of 
biodegradable plastics and the recycling path that could be used was performed in order to compare this technology to the first two processes.

Pyrolysis and gasification are energy intense chemical processes, however they offer the the ability to be automated, ruggidized and have similarities to oxygen production proccesses intended for lunar applications. Biological processes are lower in energy but require environmental controls to keep the bacterial colonies in balance. Further work on all of the technologies is needed in order to more accurately compare the processes and determine the optimal choice for lunar applications.

Experimental work was performed to investigate the distribution of products at varying temperature and gas compositions. Two experimental approaches were taken to examine the degradation products using a tube furnace as the heated chamber. A flow through system was set up to deliver the degradation products from the furnace to gas sampling valves on a GC-MS (Figure 1). Samples were taken approximately every 35 seconds and the degradation products were monitored over a 30 minute period. The carrier gas fed into the furnace to deliver the products to the gas sampling valves had a total flow of $30 \mathrm{sccm}$ of gas, consisting of either helium or helium and a varying percentage of oxygen depending on the experiment being run (pyrolysis or gasification). Three sampling tempeartures were chosen to evaluate, $400 \mathrm{C}, 600 \mathrm{C}$ and $800 \mathrm{C}$. This range was chosen based on the STA data, showing degradation starting at 400C. 600 C was chosen as the next temperature because it was below the melting point of aluminum, a component in some of the sample bags. Avoiding the melting of aluminum maximizes the oxygen conversion to $\mathrm{CO}$ and $\mathrm{CO}_{2}$. Melting the aluminum would expose unoxidized aluminum which would react with the oxygen in the reaction chamber. These side reactions of oxygen need to be minimized because the oxygen is a limited resource on the lunar surface. $800 \mathrm{C}$ was chosen as the highest temperature because it was comparable to temperatures used in other high temperature lunar technologies (hydrogen reduction).

The samples at $400 \mathrm{C}$ had limited products, mainly methane/ethane and $\mathrm{CO} / \mathrm{CO}_{2}$ for pyrolysis and gasification, respectively. Higher temperatures in pyrolysis showed new products being produced. Higher moleculat weight hydrocarbons were produced at higher temperatures. $800 \mathrm{C}$ showed the largest amount of hydrocarbon production with several cyclic molecules being produced. Although these samples showed differences between the samples at different temperatures and different gas compositions, it was unclear how we could clearly identify the temperature effects during the ramp up phase of the experiment. The problem was that there was gas swept into the detector while the polymer/furnace was heating, so some of the gas being analyzed was produced when the sample was at a lower temperature during ramping. 


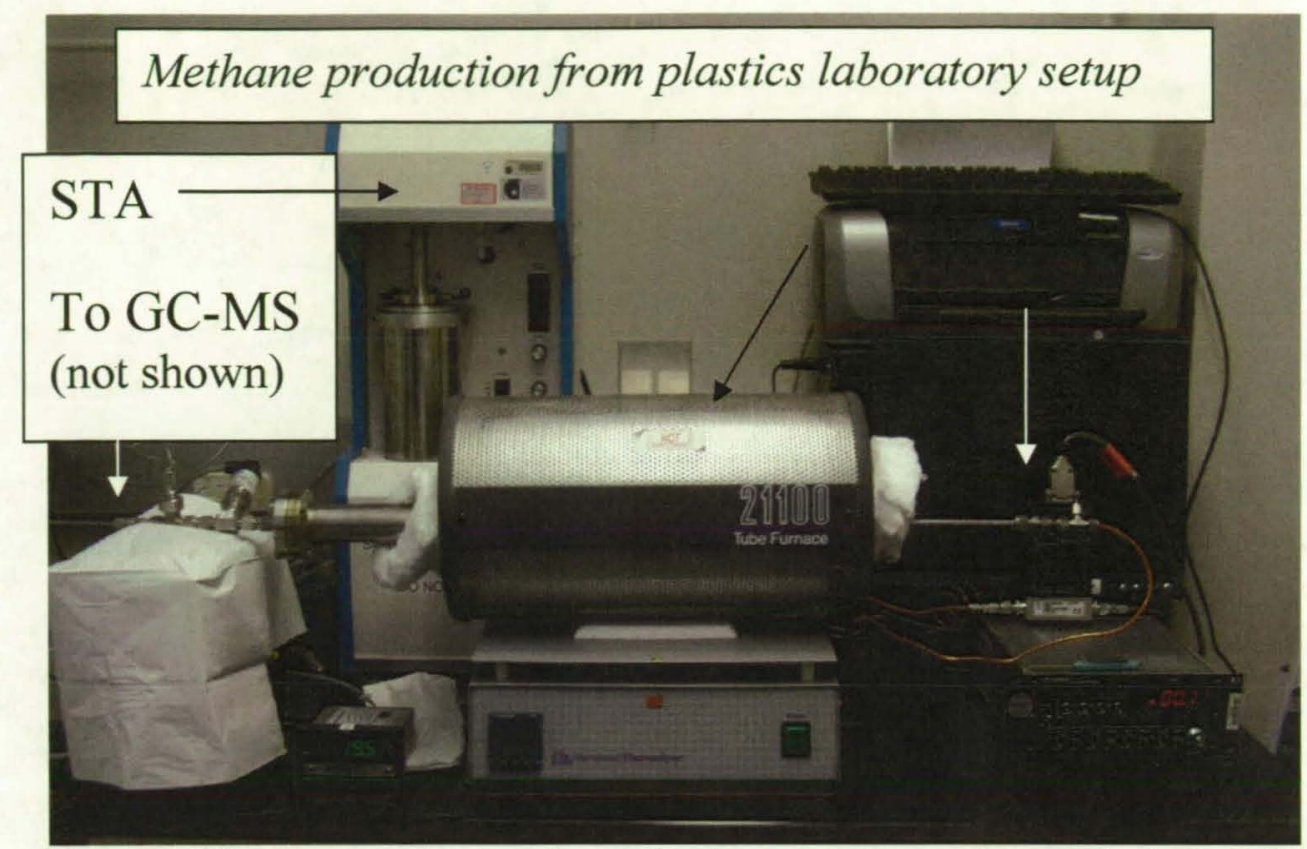

Figure 1 Methane production from plastics experimental setup including STA, Furnace, and Mass Flow Controllers

A static system was then developed to increase the residence time of the products in the heated zone at the desired temperature. Valves were added to isolate the heates zone with a pressure transducer to monitor the pressure. The samples were heated for 30-35 minutes in a static (no flow) system. The system pressure was monitored during the heating to measure the gas evolved during the degradation of the polymer. The valves isolating the heated zone were opened and an inert purge gas was used to deliver the sample to the GC-MS. The products were again analyzed by the GC-MS however only 1-2 samples were taken and chromatography was performed for separation of $\mathrm{CO}_{2}$ from the inert gasses. The $\mathrm{GC}$ program ramped the temperature from $25 \mathrm{C}$ to $300 \mathrm{C}$ with a total run time of 13 minutes. This system more accurately mimics the system that would be employed on the lunar surface although it is not perfect. There are colder areas that are outside the furnace but included in the isolated volume. This is due to the fact that we do not have high temperature pressure transducers and valves so they could not be in the heated zone. Figure 2 shows the spectra of two samples comparing pyrolysis and gasification at $800 \mathrm{C}$. During gasification, there are two peaks, one from the inert gases and a second from $\mathrm{CO}_{2}$. The products of pyrolysis are much more complicated starting from methane and continuing up to benzene and other complex hydrocarbons. 


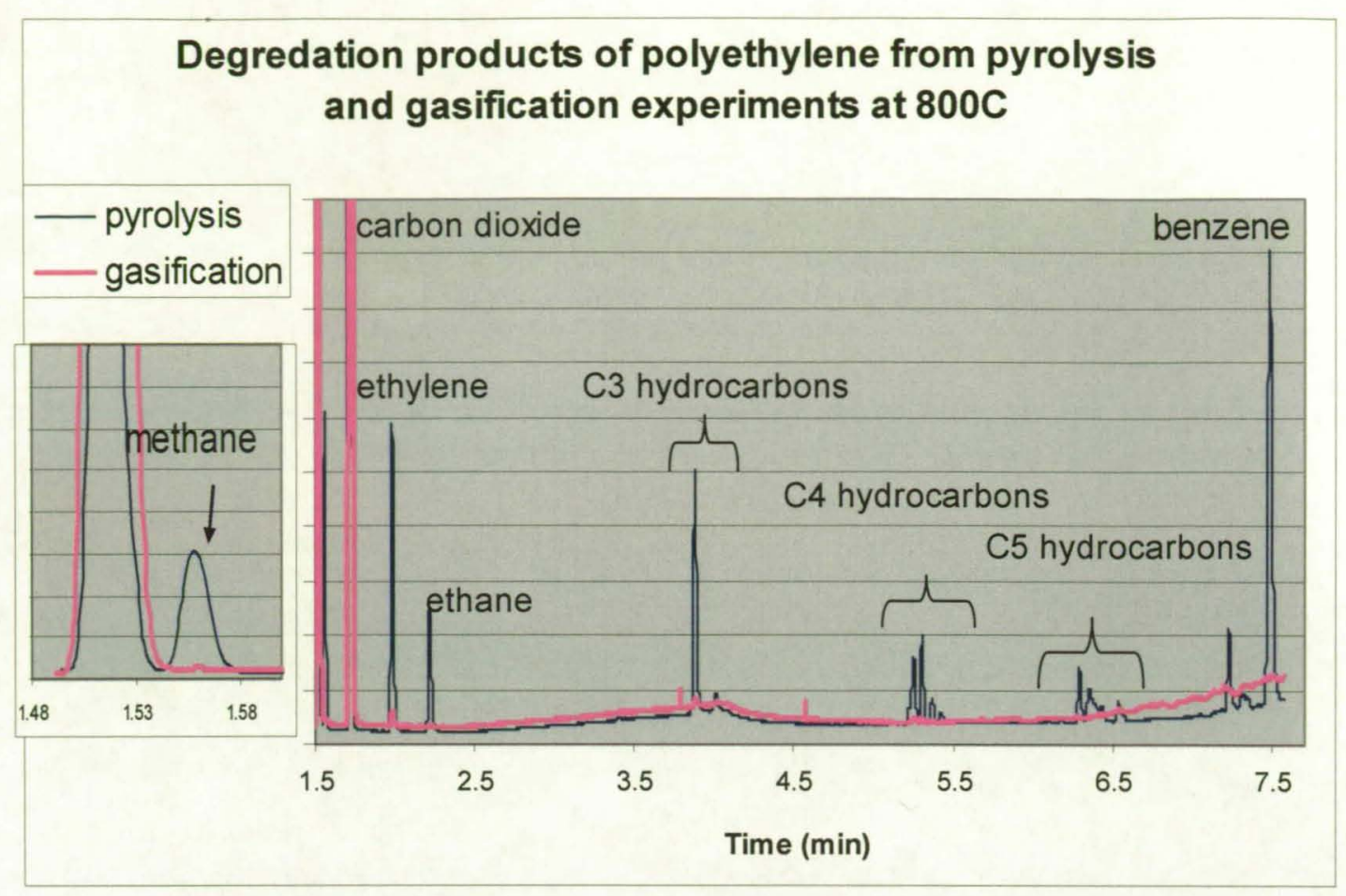

Figure 3 shows the $\mathrm{CO}-\mathrm{CO}_{2}$ relative peak areas during the flow through experiments at $600 \mathrm{C}$ with varying concentrations of oxygen. The trend in Fig. 3 suggests that the production of $\mathrm{CO}-\mathrm{CO}_{2}$ is proportional with the oxygen concentration in the gas phase.

During the static experiments, the amount of $\mathrm{CO}_{2}$ produced saturated the detector at mass 44 which did not allow us to compare the amount of $\mathrm{CO}_{2}$ for the varying runs. A dilution system was set up to dilute the sample $15: 1$, taking the $\mathrm{CO}_{2}$ down to a detectable level. The dilution runs were conducted at $400 \mathrm{C}, 600 \mathrm{C}$ and $800 \mathrm{C}$ with an atmosphere consisting of $20 \%$ oxygen and $80 \%$ helium. At $400 \mathrm{C}$, there is no appreciable $\mathrm{CO}_{2}$ measured during the diluted sample runs $(\sim 1 \%)$. The highest concentration of $\mathrm{CO}_{2}$ was measured from the samples at $600 \mathrm{C}$. The samples at $800 \mathrm{C}$ had lower levels of $\mathrm{CO}_{2}$ than the samples at $600 \mathrm{C}$, most likely due to the increase in the formation of hydrocarbons, benzene, tolune, etc.

\section{Degradation of polyethylene at $600 \mathrm{C}$ with different oxygen concentrations}

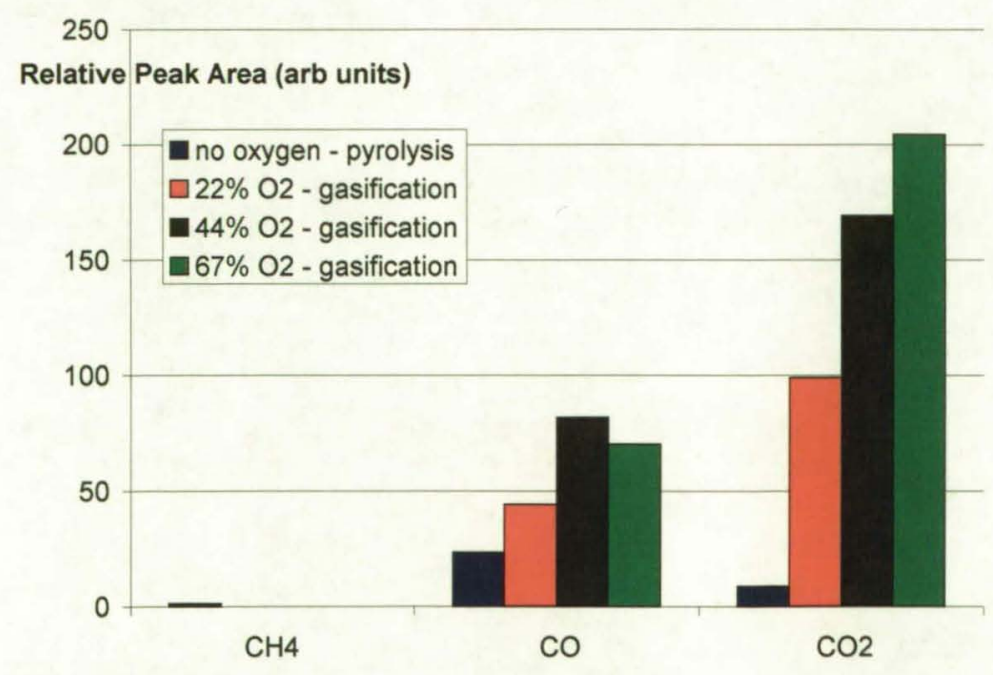

This data led us to the conculsion that $600 \mathrm{C}$ with oxygen is the most favorable condition to set up a demonstration unit for methane production. Although the amount of oxygen could be varied to further optimize the system, limited time and funding has limited our ability to fully characterize all of the varaibles in our system. A benchtop system for 
processing plastics is being designed for a prototype demonstration. This breadboard unit will convert plastics into methane using a furnace for degradation and a Sabatier reactor for conversion of carbon dioxide into methane, providing valuable information on system design trades that will improve the processes efficiency and automation.

References

1. Final Report Production of Methane and Water From Plastics and Crew Waste, C Parrish Consulting, Order Number NNK07EA25P

2. CxP 70024 Baseline Constellation Program Human-Systems Integration Requirements, Release Date 12/15/06

3. ESCG-4470-07-TEAN-DOC-0204 Exploration Life Support Reference Missions

Document Bruce E. Duffield, Jacobs Technology, Inc., January 2008 